\title{
LESIÓN DEL NERVIO CUBITAL SECUNDARIO A TERAPIA DE ONDAS DE CHOQUE EXTRACORPÓREAS RADIALES IDENTIFICADA CON ULTRASONOGRAFÍA DE ALTA RESOLUCIÓN: REPORTE DE CASO
}

\author{
ULNAR NERVE INJURY AFTER RADIAL EXTRACORPOREAL SHOCK WAVE THERAPY \\ IDENTIFIED WITH HIGH-RESOLUTION ULTRASONOGRAPHY: CASE REPORT \\ Paul Terán-Vela ${ }^{1, a}$, Walter Insuasti-Abarca², Diana Martínez-Asnalema ${ }^{3}$, Tania Platero-Portillo4, \\ Sebastián Ramos-Rosas 5,b, Sussan Llocclla-Delgado ${ }^{6}$
}

\begin{abstract}
RESUMEN
Mujer de 49 años fue evaluada por desarrollar dolor súbito a nivel de los epicóndilos mediales conjuntamente con entumecimiento y sensación de hormigueo en el cuarto y quinto dedos después de recibir terapia de ondas de choque extracorpóreas radiales (rESWT) como tratamiento para epicondilitis medial bilateral. EI examen neurológico reveló signo de Tinel positivo, parestesia y una prueba de discriminación de dos puntos alterada sobre la región cubital del cuarto y quinto dedos. La ultrasonografía de alta resolución demostró hallazgos de lesión nerviosa periférica tales como hipoecogenicidad y aumento del diámetro de ambos nervios cubitales. La paciente mejoró únicamente tras recibir tratamiento conservador, demostrando así una aparente neuropatía compresiva bilateral aguda como resultado de la terapia. ESWT se utiliza como una alternativa prometedora para el tratamiento de diversos trastornos musculoesqueléticos; sin embargo, existe evidencia limitada con respecto a sus efectos secundarios, en particular la mononeuropatía periférica. Según el conocimiento de los autores, este es el primer reporte que demuestra un daño nervioso estructural después de rESWT con el uso de ultrasonografía de alta resolución.
\end{abstract}

Palabras clave: Neuropatía cubital; Terapia de ondas de choque extracorpóreas; Epicondilitis medial; Ecografía; Reporte de caso (fuente: DeCS BIREME).

\begin{abstract}
A 49-year old woman was evaluated for developing bilateral acute medial elbow pain, numbness, and tingling sensation in the fourth and fifth fingers after receiving radial extracorporeal shock wave therapy (rESWT) for bilateral medial epicondylitis. Neurologic examination revealed the presence of Tinel's sign, paresthesia and impaired two-point discrimination testing over the ulnar side of the fourth and fifth fingers bilaterally. High-resolution ultrasonography demonstrated findings of nerve injury, such as hypoechogenicity and increased diameter of both ulnar nerves. After conservative treatment, the patient improved her condition demonstrating an apparently acute compressive nerve injury as a result of the therapy. ESWT is used as a promising alternative for the treatment of various musculoskeletal disorders; however, there is limited evidence regarding its side effects, in particular peripheral mononeuropathy. To the authors' knowledge, this is the first report demonstrating structural damage of a nerve after rESWT with the use of high-resolution ultrasonography.
\end{abstract}

Key words: Ulnar neuropathy; Extracorporeal shockwave therapy; Medial epicondylitis; Ultrasonography; Case report (source: MeSH NLM).

\footnotetext{
${ }^{1}$ Centro de Especialidades Ortopédicas, Quito-Ecuador

${ }^{2}$ Departamento de Medicina Interna, Centro de Especialidades Ortopédicas, Quito-Ecuador.

${ }^{3}$ Departamento de Medicina Física y Rehabilitación, Hospital General Luis Dávila, Tulcán-Ecuador

${ }^{4}$ Escuela de Medicina, Universidad de El Salvador, San Salvador-El Salvador.

${ }^{5}$ Centro Médico Ramos-Vera, Quito-Ecuador.

${ }^{6}$ Instituto de Investigaciones Biomédicas. Universidad Ricardo Palma, Santiago de Surco-Perú.

${ }^{a}$ Cirujano ortopédico, ${ }^{\text {b }}$ Máster en salud pública.

Citar como: Paul Terán-Vela, Walter Insuasti-Abarca, Diana Martínez-Asnalema, Tania Platero-Portillo, Sebastián Ramos-Rosas, Sussan Llocclla-Delgado. Lesión del nervio cubital secundario a terapia de ondas de choque extracorpóreas radiales identificada con ultrasonografía de alta resolución: reporte de caso. Rev. Fac. Med. Hum. Abril 2020; 20(2):328-333. DOI 10.25176/RFMH.v20i2.2912
} 


\section{INTRODUCCIÓN}

La epicondilitis medial es un trastorno común que afecta la extremidad superior. A pesar de una prevalencia general de $<1 \%$, la epicondilitis medial puede afectar entre $3,8 \%$ a $8,2 \%$ de pacientes en entornos ocupacionales ${ }^{(1)}$.

Aunque la terapia no quirúrgica y el tratamiento quirúrgicoabiertoson los pilares del tratamientoparaesta patología, estudios recientes han evaluado el impacto de las terapias no tradicionales como la terapia de ondas de choque extracorpóreas (ESWT) mostrando resultados prometedores ${ }^{(1,2)}$; sin embargo, existe evidencia limitada con respecto a sus efectos secundarios, en particular la mononeuropatía periférica. Según el conocimiento de los autores, este es el segundo caso que informa una lesión nerviosa periférica después de la aplicación de terapia de ondas de choque extracorpóreas para la epicondilitis medial(3), y el primero que demuestra un daño estructural del nervio con el uso de ultrasonografía de alta resolución. Además, este reporte intenta aclarar los posibles mecanismos fisiopatológicos involucrados en la lesión del nervio cubital debido a la terapia de ONDAS de choque extracorpóreas radiales (rESWT).

\section{CASO CLÍNICO}

Una mujer previamente sana de 49 años llegó a nuestra clínica por presentar dolor a nivel de los epicóndilos mediales en ambos brazos de un año de evolución. La paciente es cocinera y generalmente mueve objetos pesados de un lado a otro. Inicialmente, fue vista en otro centro médico y diagnosticada con epicondilitis medial bilateral, por lo que se le prescribieron varias sesiones de fisioterapia que no mejoraron su condición. Después de una segunda evaluación en el mismo centro, le prescribieron siete sesiones de terapia de ondas de choque extracorpóreas radiales (rESWT) en un intervalo de una por semana. Según la paciente, después de la tercera sesión de rESWT, sintió entumecimiento y sensación de hormigueo en el cuarto y quinto dedos de forma bilateral, síntomas que no se percibían antes. Además, la intensidad del dolor aumentó al punto que la inhabilitó para extender los codos, por lo que decidió no seguir recibiendo más sesiones de rESWT.
Tres meses después de la última sesión de rESTW, la paciente fue derivada a nuestra clínica. En el examen físico, no pudo extender sus codos más allá de los 90 grados de flexión debido a un dolor insoportable (10/10 en la Escala Visual Analógica). El dolor y la hinchazón sobre los músculos epicóndilos mediales fueron evidentes. El examen neurológico reveló la presencia del signo de Tinel, parestesia y una prueba de discriminación de dos puntos alterada sobre el lado cubital del cuarto y quinto dedos bilateralmente. Las radiografías simples de ambos codos eran normales. La elastografía porultrasonido mostróinflamaciónyfibrosis sobre la inserción de los tendones flexores comunes en los epicóndilos mediales (Figura 1). La ultrasonografía de alta resolución demostró hipoecogenicidad y un aumento del diámetro de ambos nervios cubitales (Figura 2). Finalmente, los estudios electrodiagnósticos no mostraron evidencia de alteraciones del nervio cubital o mediano, y los análisis de sangre mostraron una dislipidemia leve.

La primera actitud terapéutica fue aconsejar a la paciente que limite las actividades repetitivas de flexión de muñeca y pronación del antebrazo, además de evitar levantar y manipular objetos pesados. Asimismo, se prescribieron medicamentos analgésicos, cambios en el estilo de vida, dieta y modalidades de fisioterapia como ultrasonido, TENS, ejercicios que mejoren el rango de movimiento y estiramientos.

Además, se aplicaron 10 sesiones de laser de alta intensidad (HIL) a día seguido con protocolo: Analgesia: dosis: $10 \mathrm{~J} / \mathrm{cm}^{2}$ en área de $10 \mathrm{~cm}^{2}$ y potencia $5,00 \mathrm{~W}$. Bioestimulación: dosis $60 \mathrm{~J} / \mathrm{cm} 2$ en área de $10 \mathrm{~cm}^{2}$ y potencia $5,00 \mathrm{~W}$.

Después de dos meses, la paciente mejoró considerablemente sus síntomas. La paciente pudo extender sus codos y el dolor casi se resolvió (2/10 en la escala analógica visual). El examen neurológico y las pruebas de función motora fueron normales.

El cuestionario sobre las discapacidades del hombro, codo y mano (DASH) medidos antes y después del tratamiento conservador fueron $A: 60,34, B: 50, C: 81,25$ y A: $12,06, B: 25, C: 25$, respectivamente. La paciente continuó recibiendo fisioterapia con introducción gradual y progresión de ejercicios de fuerza excéntrica. 


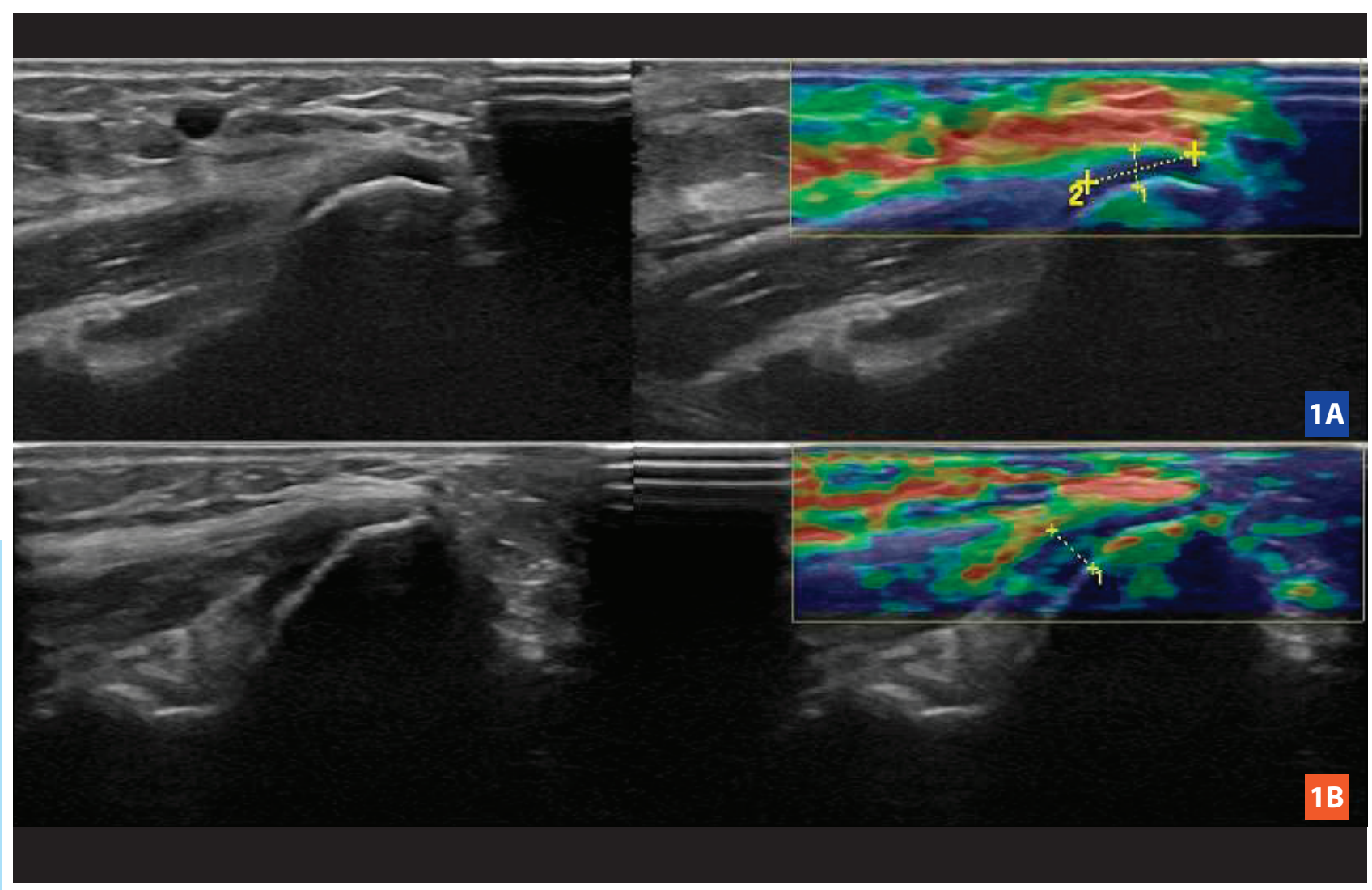

Figura 1. La elastografía por ultrasonido muestra inflamación y fibrosis sobre la inserción de los tendones flexores comunes en los epicóndilos mediales derecho (1A) e izquierdo (1B).

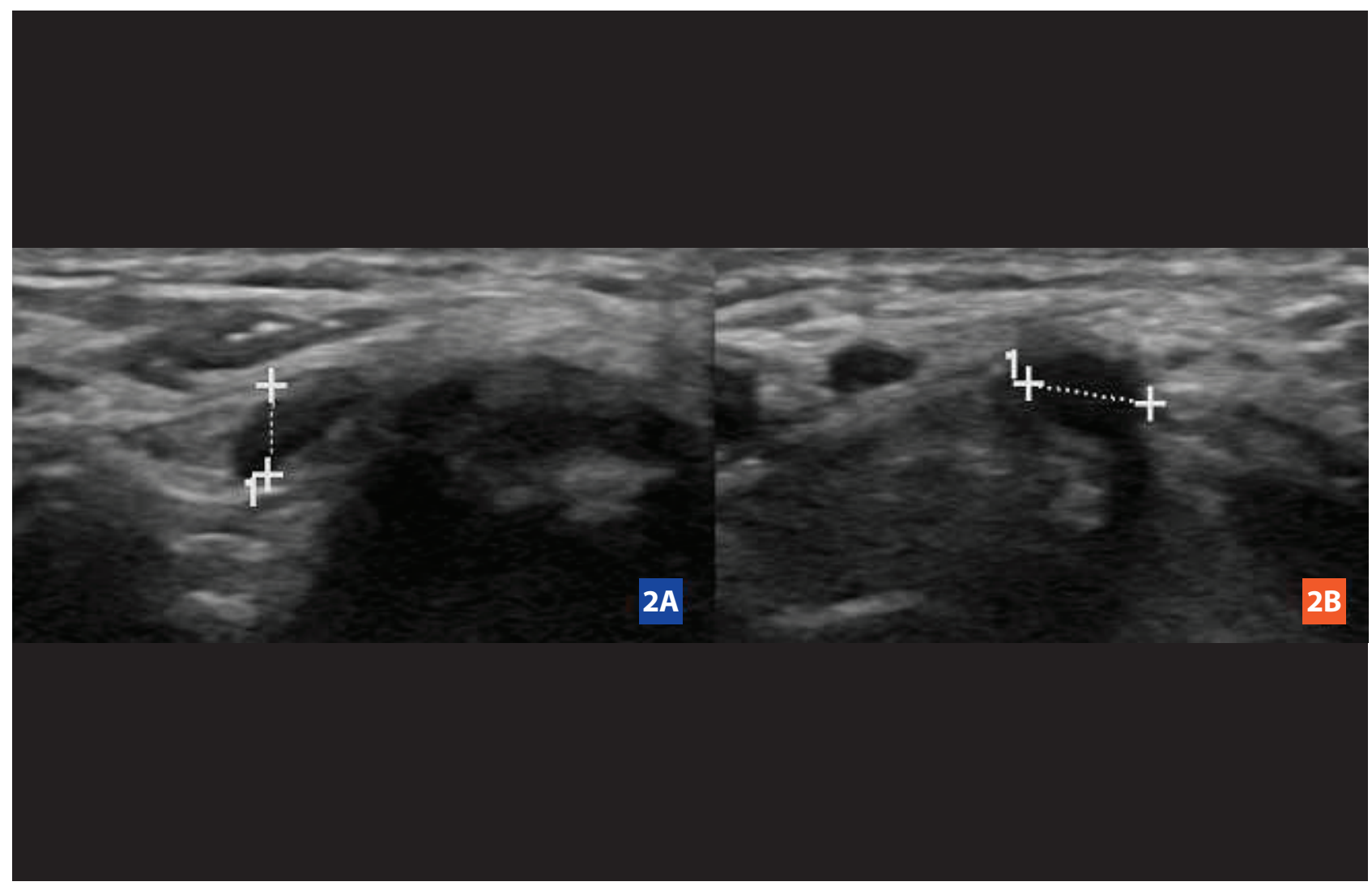

Figura 2. La ultrasonografía de alta resolución muestra hipoecogenicidad y un diámetro aumentado $(4,5 \mathrm{~mm})$ de los nervios cubitales derecho (2A) e izquierdo (2B). 


\section{DISCUSIÓN}

Múltiples pruebas apoyan el uso de la terapia de ondas de choque extracorpóreas (ESWT) como efectiva y segura en el tratamiento de varios trastornos musculoesqueléticos ${ }^{(4)}$, y a pesar que no se pueden realizar recomendaciones definitivas para el uso de ESWT para la epicondilitis medial en este momento, ${ }^{(1,5)}$ la Sociedad Internacional para el Tratamiento Médico de Ondas de Choque (ISMST) ha reconocido su uso clínico en esta tendinopatía(6). Además, Lee et al. ${ }^{(7)}$ demostraron la eficacia de la ESWT para pacientes recién diagnosticados con epicondilitis lateral o medial en comparación con la inyección local de esteroides. Sus resultados confirmaron que el grupo ESWT mejoró tanto como el grupo de inyección local de esteroides; por lo tanto, el estudio concluyó que ESWT puede ser una opción de tratamiento útil en pacientes para quienes la inyección local de esteroides es problemática. Igualmente, Turhan et al. ${ }^{(8)}$, en un análisis retrospectivo de 36 pacientes con diagnóstico de epicondilitis medial aislada refractaria a medidas conservadoras, encontraron que la ESWT era una buena opción de tratamiento conservador al mejorar significativamente las puntuaciones VAS y Q-DASH $(p<0,001)$ luego de 1 y 12 semanas después del tratamiento.

Por otra parte, con respecto a los efectos secundarios de la ESWT, en el contexto de un estudio aleatorizado, controlado con placebo, simple ciego y multicéntrico para evaluar la eficacia de la ESWT en la epicondilitis lateral, Haake et al. ${ }^{(9)}$ registraron sistemáticamente los efectos adversos de la terapia. En general, se analizaron 399 casos de ESWT, y los efectos adversos más frecuentes incluyeron enrojecimiento transitorio de la piel $(21,1 \%)$, dolor $(4,8 \%)$ y hematomas pequeños $(3,0 \%)$. De manera similar, una revisión de la literatura que involucró a 631 pacientes que se sometieron a ESWT para el tratamiento de no-uniones óseas, mostró que los efectos secundarios más comunes asociados con la terapia incluyeron equimosis leve, petequias, hematomas, inflamación leve y enrojecimiento temporal de la piel y, lo que es más importante, todos estos efectos secundarios generalmente se pudieron evitar con una orientación precisa, un posicionamiento adecuado y un estricto cumplimiento del protocolo de tratamiento ${ }^{(10)}$.

Por lo tanto, es digno de mención que, además de los efectos secundarios comunes mencionados anteriormente, ESWT podría estar asociado a daño nervioso como se observa en esta paciente. Aparentemente, las lesiones nerviosas debidas a ESWT son extremadamente infrecuentes y rara vez se informan. Una revisión de la literatura advierte solo un caso de neuropatía cubital en el codo con axonotmesis severa después de ESWT local ${ }^{(3)}$. En este reporte de caso, ESWT se aplicó a nivel del codo mostrando evidencia clínica y electrodiagnóstica de lesión nerviosa directa la cual mejoró parcialmente tres meses después de la liberación del túnel cubital con epicondilectomía medial.

A pesar de que puede observarse neuritis cubital concomitante en hasta el $20 \%$ de los pacientes con epicondilitis medial ${ }^{(11)}$, la historia y la evolución de la enfermedad de esta paciente hacen que los autores planteen la hipótesis de una posible lesión directa del nervio cubital como consecuencia de la rESWT.

Como se describe en la historia clínica, después de la tercera sesión de rESWT, la paciente experimentó un dolor insoportable, así como una sensación de entumecimiento y hormigueo en los dedos cuarto y quinto que no se percibían antes. Inicialmente, nuestro protocolo de tratamiento para el paciente consistió exclusivamente en analgesia y descanso, lo que gradualmente mejoró sus síntomas. Estos hallazgos respaldan la hipótesis de que se produjo una lesión compresiva aguda del nervio, ya que, si fuera el caso de una neuropatía cubital crónica concomitante, la paciente no habría desarrollado síntomas agudos ni mucho menos hubiese existido una recuperación espontánea ${ }^{(12,13)}$. Las compresiones nerviosas crónicas son generalmente condiciones de deterioro progresivo que persisten sin una intervención adecuada. Sus síntomas pueden comenzar con parestesia y entumecimiento distal, pero a diferencia de las compresiones agudas, a menudo progresan con el tiempo a debilidad muscular y emaciación ${ }^{(12)}$, que sin duda no fue el caso de esta paciente.

Además, vale la pena mencionar que, como la mayoría de las compresiones de nervios periféricos se consideran neuropraxias, los autores esperaban una recuperación espontánea de la paciente en un período prudencial, la cual finalmente se evidenció.

La etiología de las lesiones de compresión nerviosa aguda es a menudo evidente, ya que son el resultado de un solo episodio traumático ${ }^{(13)}$.

Además, es importante resaltar que los mecanismos por los cuales la ESWT podría causar daño nervioso aún son inciertos. Hausdorf et al. ${ }^{(14)}$ observaron la destrucción selectiva de fibras nerviosas no mielinizadas dentro de los nervios femorales de conejos posteriores a ESWT de alta energía. Del mismo modo, Schelling et al. ${ }^{(15)}$ mostraron en un estudio in vitro de nervios ciáticos de rana que los efectos biológicos de las ondas de choque en el tejido nervioso parecen ser el resultado de la cavitación. Como resultado, al igual que Shim et al.(3) hipotetizaron, un posible mecanismo de lesión nerviosa podría ser la transmisión de energía precipitada de la onda de choque por el efecto de cavitación producido por la acumulación de líquido en la epicondilitis medial. 
Otro mecanismo para la lesión del nervio cubital después de la rESWT podría ser la compresión aguda del nervio debido a la inflamación adyacente de los tejidos blandos como se ve regularmente en el traumatismo del codo ${ }^{(16)}$. Para recapitular, los efectos secundarios reconocidos de la ESWT incluyen hinchazón y pequeños hematomas ${ }^{(9,10)}$; en consecuencia, ESWT podría haber desencadenado o exacerbado la inflamación regional alrededor del epicóndilo medial y así comprimir el nervio cubital adyacente ${ }^{(3)}$.

Normalmente, el efecto de ESWT en cualquier otra área de aplicación habría sido inofensivo; sin embargo, dado que el sitio de aplicación de ESWT para la epicondilitis medial es un área de alto riesgo de atrapamiento para el nervio cubital mientras viaja profundamente bajo el ligamento de Osborne ${ }^{(17)}$, la hinchazón y la inflamación subyacente podrían haber provocado una lesión compresiva aguda e iniciar una respuesta biológica que incluye el engrosamiento de las paredes del endoneuro y microvasos perineurales, engrosamiento del endoneuro como resultado de edema, un aumento en la cantidad de tejido conectivo; y más tarde, engrosamiento del epineuro y perineuro debido a fibrosis y edema. Finalmente, estas características fisiopatológicas que llevaron a afectar los potenciales de acción y el transporte axonal ${ }^{(18,19,20,21)}$ se evidenciaron gracias a la ultrasonografía.

Varios estudios han validado la importancia de la ecografía neuromuscular para el diagnóstico de trastornos de nervios periféricos ${ }^{(18,19,20,21)}$. Dado que el engrosamiento del nervio es el indicador de diagnóstico más confiable de un nervio anormal, la medición del tamaño del nervio es crítica ${ }^{(20)}$. Los hallazgos ecográficos en este paciente revelaron un diámetro del nervio de 4,5 mm. Con relación a esto, Beekman et al. ${ }^{(19)}$ demostraron que los pacientes con neuropatía cubital en el codo tenían un mayor diámetro del nervio cubital que los controles ( $p<0,0001)$. Los valores de referencia para las medidas ecográficas demostraron que un diámetro del nervio cubital mayor de $2,5 \mathrm{~mm}$ a nivel del epicóndilo medial se consideraba anormal. Los signos adicionales de lesión compresiva del nervio consisten en engrosamiento local, hipoecogenicidad con pérdida de su patrón fascicular, característica que también se evidenció en esta paciente, e hipervascularización intraneural en Doppler color o power ${ }^{(20,21)}$.

Finalmente, es imperativo mencionar el hecho de que los estudios electrodiagnósticos en este caso fueron negativos. El electrodiagnóstico sigue siendo el estándar de oro para la neuropatía del nervio cubital ${ }^{(18)}$. Sin embargo, el rendimiento diagnóstico es menor, y las interpretaciones de los datos a menudo son más difíciles ${ }^{(22)}$, ya que su sensibilidad varía del 37 al $86 \%{ }^{(19)}$. Por lo tanto, diferentes estudios ${ }^{(18,19,20,23)}$ han sugerido un enfoque multidisciplinario para el diagnóstico del síndrome de compresión del nervio cubital a través de análisis de signos clínicos en combinación con estudios electrodiagnósticos y de imagen, ya que incluso en pacientes con compresión clínica evidente del nervio cubital, la electromiografía y los estudios de conducción nerviosa pueden obtener resultados falsos negativos en hasta el $10 \%$ de los pacientes ${ }^{(24)}$.

Para explicar la alta incidencia de resultados falsos negativos, la evidencia respalda los errores técnicos como la principal fuente de diagnósticos erróneos ${ }^{(25)}$. Otra posible explicación de un resultado falso negativo en este caso podría estar relacionado con el tiempo transcurrido desde la lesión nerviosa hasta el momento que se realizó el estudio electrodiagnóstico.

Como consecuencia, es importante resaltar el uso del ultrasonido en este caso. Primero, la sensibilidad del área transversal o diámetro aumentados del nervio cubital en el codo es más del $80 \%$ en la neuropatía cubital en el codo. Además, la ecografía a veces puede identificar el sitio de atrapamiento en pacientes con sospecha de neuropatía cubital en el codo que se presentan con resultados normales en las pruebas electrodiagnósticas ${ }^{(20,26,27)}$.

Una limitación a considerar es la naturaleza retrospectiva de este reporte. Como la paciente recibió rESWT en otro centro médico, no pudimos recopilar información valiosa sobre el protocolo de tratamiento, incluida la dosis de energía utilizada, o el sitio preciso de la aplicación de rESWT.

Además, aparentemente la terapia fue aplicada por profesionales que no estaban certificados porla Sociedad Internacional para el Tratamiento Médico de Ondas de Choque (ISMST) o por la Federación Iberoamericana de Sociedades y Asociaciones de Ondas de Choque en Medicina (ONLAT). Como resultado, algunos requisitos específicos necesarios para garantizar una técnica de aplicación adecuada, minimizar posibles efectos adversos y mejorar la seguridad del paciente podrían no haberse cumplido.

\section{CONCLUSIÓN}

Este caso demuestra los efectos potencialmente dañinos de la aplicación de rESWT para la epicondilitis medial. Como se indicó anteriormente, es evidente que diferentes mecanismos fisiopatológicos podrían haber estado involucrados en el desarrollo de una lesión aguda del nervio cubital en esta paciente.

Finalmente, es imperativo resaltar el uso de la ultrasonografía de alta resolución en este caso ya que nos permitió corroborar el diagnóstico de neuropatía cubital debido a rESWT en el contexto de hallazgos electrodiagnósticos negativos. 
Contribuciones de autoría: Los autores realizaron la génesis de la idea, diseño de proyecto, recolección e interpretación de datos, análisis de resultados y preparación del manuscrito del presente trabajo de investigación.

Financiamiento: Autofinanciado.

Correspondencia: Walter Insuasti.

Dirección: Av.Miguel Angel y Calle de Las Avellanas, Quito-Ecuador.

Teléfono: Ph: 593 (02)4503252 - 2267490 - 2267491

Correo:walter_insuasti@hotmail.com
Conflicto de interés: Los autores declaran no tener conflicto de interés en la publicación de este artículo.

Recibido: 21 de febrero 2020

Aprobado: 17 de marzo 2020

\section{REFERENCIAS BIBLIOGRÁFICAS}

1. Amin NH, Kumar NS, Schickendantz MS. Medial epicondylitis: evaluation and management. J Am Acad Orthop Surg. 2015; 23: 348-355.

2. Moya, Daniel \& Ramon, Silvia \& Schaden, Wolfgang \& Wang, Ching-Jen \& Guiloff, Leonardo \& Cheng, Jai-Hong. The Role of Extracorporeal Shockwave Treatment in Musculoskeletal Disorders. JBJS 2018; 100: 251.

3. Shim JS, Chung SG, Bang H, Lee HJ, Kim K. Ulnar neuropathy after extracorporeal shockwave therapy: a case report. PMR 2015; 7: 667-70.

4. Wang, Ching-Jen. Extracorporeal shockwave therapy in musculoskeleta disorders. Journal of orthopaedic surgery and research. 2012; 7: 1186.

5. Dingemanse, Rudi \& Manon, Soubde \& Koes, Bart \& Huisstede, Bionka. Evidence for the effectiveness of electrophysical modalities for treatment of medial and lateral epicondylitis: A systematic review. BJSPORTS. 2013; 10: 1136.

6. Consensus Statement on ESWT Indications and Contraindications. ISMST. October 12th, 2016. https://www.shockwavetherapy.org/fileadmin/user_upload/ dokumente/PDFs/Formulare/ISMST_consensus_statement_on_indications_and_ contraindications_20161012_final.pdf. October 12th, 2016.

7. Seok Lee, Sang \& Kang, Sangkuk \& Kyoung Park, Noh \& Woo Lee, Chan \& Sup Song, Ho \& Kyun Sohn, Min \& Cho, kang Hee \& Hwan Kim, Jung. Effectiveness of Initial Extracorporeal Shock Wave Therapy on the Newly Diagnosed Lateral or Medial Epicondylitis. Annals of rehabilitation medicine. ARM. 2012; 36: 681.

8. Turhan, Yalcin \& Arican, Mehmet \& Okan Karaduman, Zekeriya. Clinical and functional outcomes of extracorporeal shock wave therapy in isolated medial epicondylitis. The European Research Journal 2019; 5(4): 658-662.

9. M, Haake \& R Böddeker, I \& T, Decker \& M, Buch \& M, Vogel \& G, Labek \& Maier Maresa \& Loew, Markus \& O, Maier-Boerries \& J, Fischer \& Betthäuser, A \& C Rehack H \& W, Kanovsky \& Müller, I \& Gerdesmeyer, Ludger \& Rompe, Jan. Side-effects of extracorporeal shock wave therapy (ESWT) in the treatment of tennis elbow. Archives of orthopaedic and trauma surgery. 2002; 10: 1007.

10. Furia, John \& Rompe, Jan \& Cacchio, Angelo \& Maffulli, Nicola. Shock wave therapy as a treatment of nonunions, avascular necrosis, and delayed healing of stress fractures. Foot and ankle clinics 2010; 15(4): 651-662.

11. B Barco, Raul \& Antuña, Samuel. Medial elbow pain. EFORT Open Rev. 2017; 2 (8): 362-371.

12. Menorca RMG, Fussell TS, Elfar JC. Peripheral nerve trauma: mechanisms of injury and recovery. Hand Clin. 2013; 29: 317-330.

13. Tapadia, Minal \& Mozaffar, Tahseen \& Gupta, Ranjan. Compressive Neuropathies of the Upper Extremity: Update on Pathophysiology, Classification, and Electrodiagnostic Findings. The Journal of hand surgery. 2010; 35: 668-77.
14. Hausdorf J, Lemmens MA, Heck KD, et al. Selective loss of unmyelinated nerve fibers after extracorporeal shockwave application to the musculoskeletal system. Neuroscience 2008; 155: 138-144.

15. Schelling G, Delius M, Gschwender M, Grafe P, Gambihler S. Extracorporeal shock waves stimulate frog sciatic nerves indirectly via a cavitation-mediated mechanism. Biophys J 1994; 66: 133-140.

16. Dy C, Mackinnon S. Ulnar neuropathy: Diagnosis and management. Curr Rev Musculoskelet Med. 2016; 9: 178-84

17. Macchi, Veronica \& Tiengo, Cesare \& Porzionato, Andrea \& Stecco, Carla \& Sarasin, Gloria \& Tubbs, R. Shane \& Maffulli, Nicola \& De Caro, Raffaele. The cubital tunnel: A radiologic and histotopographic study. Journal of anatomy. JOA. 2014; 10: 225

18. Campbell WW, Carroll C, Landau ME. Ulnar neuropathy at the elbow. Five new things Neurology. 2015; 5: 135-141.

19. Beekman, Roy \& Schoemaker, Mc \& P L Van Der Plas, J \& H Van Den Berg, L \& Franssen, H \& Wokke, J.h.j \& M J Uitdehaag, B \& Visser, Leo H. Diagnostic value of high-resolution sonography in ulnar neuropathy at the elbow. Neurology. 2004; 62: 767-73.

20. Suk Jl, Walker FO, Cartwright MS. Ultrasonography of peripheral nerves. Curr Neurol Neurosci Rep. 2013; 13: 328

21. Deniel, A \& Causeret, A \& Moser, Thomas \& Rolland, Yan \& Dréano, T \& Guillin, Raphaël. Entrapment and traumatic neuropathies of the elbow and hand: An imaging approach. Diagnostic and Interventional Imaging. 2015; 10: 1006.

22. E Landau, Mark \& W Campbell, William. Clinical Features and Electrodiagnosis of Ulnar Neuropathies. Physical medicine and rehabilitation clinics of North America. 2013; 24: 49-66

23. Pais $P$, Fodor $D$, Fisher T. Bilateral cubital tunnel syndrome: Case report with review of the literature. Med Ultrasonogr. 2009; 11:31-36.

24. Navarro E. Síndromes compresivos del nervio cubital en codo y muñeca. Medigraphic org. 2014; 10: 26.

25. Checkles NS, Russakov AD, Piero DL. Ulnar nerve conduction velocity-effect of elbow position on measurement. Arch Phys Med Rehabil 1971; 53: 362.

26. Shik Yoon, Joon \& O Walker, Francis \& Cartwright, Michael. Ulnar neuropathy with normal electrodiagnosis and abnormal nerve ultrasound. Arch Phys Med Rehabil. 2010; 91(2): 318-320.

27. Bismil QMK, Bismil MSK. Cubital tunnel syndrome with false-negative nerve conduction studies treated with wide awake ulnar nerve decompression via the OSWA pathway. OA Case Reports 2013; 12: 113. 